\title{
Generic saddle-node bifurcation for cascade second order ODEs on manifolds
}

\author{
by Milan Medveð̌ (Bratislava)
}

\begin{abstract}
Cascade second order ODEs on manifolds are defined. These objects are locally represented by coupled second order ODEs such that any solution of one of them can represent an external force for the other one. A generic saddle-node bifurcation theorem for 1-parameter families of cascade second order ODEs is proved.
\end{abstract}

1. Introduction. Consider the Duffing forced oscillator

$$
\ddot{x}+\gamma \dot{x}+\omega^{2} x+\alpha x^{3}=g(t),
$$

where $\alpha, \omega, \gamma \in \mathbb{R}$ and $g(t)$ is an external force. If $g(t)=\beta \cos k t, \beta, k \in \mathbb{R}$, then $g$ is a solution of the differential equation

$$
\ddot{y}+k^{2} y=0 .
$$

We study the control problem consisting in finding a solution $g(t)$ of equation (1.2) such that equation (1.1) has a periodic or homoclinic solution or more generally some prescribed qualitative properties. If we are able to describe the topological structure of trajectories of the system

$$
\ddot{x}+\gamma \dot{x}+\omega^{2} x+\alpha x^{3}=y, \quad \ddot{y}+k^{2} y=0
$$

then we have a chance to solve this control problem. While equation (1.1) is scalar and nonautonomous, the system (1.3) is two-dimensional, autonomous. Despite this fact it is sometimes more convenient to study autonomous systems in a higher dimensional state space than the nonautonomous ones with lower dimensional variables. Systems of the form (1.3), where the second equation is independent of the first one, are called cascade systems. In [2] we have studied nonautonomous cascade systems of second order differential equations of the form

$$
\ddot{x}+R(y, t) x=f(x, y, t), \quad \ddot{y}+S(y) y=0,
$$

1991 Mathematics Subject Classification: 35B32, 58F14.

Key words and phrases: cascade, ODE, critical element, transversal, bifurcation. 
and a sufficient condition for the oscillation of solutions was proved. Cascade systems of first order ODEs of the form

$$
\dot{y}=\Phi(y, z), \quad \dot{z}=\Psi(z, u)
$$

are studied from the point of view of their feedback stabilization $(u$ is a control parameter) in [8].

We define cascade second order ODEs on manifolds which can represent two or more coupled nonlinear oscillators controlled by a second order ODE. We study generic bifurcations of such objects. Generic properties of second order ODEs on manifolds have been studied by S. Shahshahani [9] and generic bifurcations of second order ODEs on manifolds are described in the papers $[3,4,5]$. We note that in [7] a class of vector fields on manifolds containing second order ODEs is studied from the generic point of view.

2. Generic saddle-node bifurcation. Recall the definition of second order ODEs on a smooth manifold $X$. Denote by $T X$ the tangent bundle of the manifold $X$ and by $T^{2} X=T(T X)$ the double tangent bundle of $X$ (see e.g. [1], [6]). Denote by $\Gamma_{I}^{r}(T X)$ the set of all $C^{r}$-vector fields on $T X$.

Definition 2.1 (see [1]). A vector field $\xi \in \Gamma_{I}^{r}(T X)$ is said to be a second order $O D E$ on $X$ if

$$
D \tau_{X} \circ \xi=\mathrm{id}_{T X}
$$

where $\tau_{X}: T X \rightarrow X$ is the natural projection, $D \tau_{X}: T^{2} X \rightarrow T X$ is the derivative of $\tau_{X}$ and $i d_{T X}: T X \rightarrow T X$ is the identity map on $T X$.

If $(U, \alpha)$ is a chart on $X$, then $\left(T_{\alpha}, \tau_{X}^{-1}(U)\right)$ is the natural chart on $T X$, where $T_{\alpha}\left([\gamma]_{x}\right)=\left(\alpha(x), d_{0}(\alpha \circ \gamma)(1)\right),[\gamma]_{x} \in T_{x} X$ and $d_{0}(\alpha \circ \gamma)$ is the Fréchet derivative of $\alpha \circ \gamma$ at $0 \in \mathbb{R}$ (see e.g. [1], [6]).

If the vector field $\xi \in \Gamma_{I}^{r}(T X)$ satisfies $(2.1)$ and $\xi_{\alpha}$ is its local representative in the chart $\left(T_{\alpha}, \tau_{X}^{-1}(U)\right)$ then

$$
\xi_{\alpha}(x, y)=(x, y, y, g(x, y))
$$

where $(x, y) \in \alpha(U) \times \mathbb{R}^{n}, g \in C^{r}\left(\alpha(U) \times \mathbb{R}^{n}, \mathbb{R}^{n}\right)$ (see [6]). The vector field (2.2) represents a special system of the form

$$
\dot{x}=y, \quad \dot{y}=g(x, y),
$$

which is obviously equivalent to the second order ODE on $\alpha(U)$ :

$$
\ddot{x}=g(x, \dot{x}) .
$$

REMARK. An interesting class of vector fields can be obtained if (2.1) is replaced by the condition $D \tau_{X} \circ \xi=A$, where $A: T X \rightarrow T X$ is a fiber preserving bundle endomorphism, linear on each fiber. Such vector fields are locally represented by systems of the form $\dot{x}=B(x) y, \dot{y}=g(x, y)$, where 
$B(x)$ is a matrix-valued function, not necessarily invertible for all $x$. Generic properties of such objects are studied in [7].

Definition 2.2. Let $X$ and $Y$ be smooth manifolds. By a cascade second order $O D E$ (briefly CS) of class $C^{r}$ on $X \times Y$ we mean a couple $\xi=(F, G)$, where $F: T X \times T Y \rightarrow T^{2} X \times T^{2} Y, G: T Y \rightarrow T^{2} Y$ are $C^{r}$-mappings with the following properties:

(1) $\pi_{1} \circ F_{\dot{y}}$ is a second order ODE on $X$ for all $\dot{y} \in T Y$, where $\pi_{1}$ : $T^{2} X \times T^{2} Y \rightarrow T^{2} X$ is the natural projection and $F_{\dot{y}}: T X \rightarrow T^{2} X \times T^{2} Y$, $F_{\dot{y}}(\dot{x}):=F(\dot{x}, \dot{y})$.

(2) $G$ is a second order ODE on $Y$ and $\pi_{2} \circ F(\dot{x}, \dot{y})=G(\dot{y})$ for all $(\dot{x}, \dot{y}) \in T X \times T Y$, where $\pi_{2}: T^{2} X \times T^{2} Y \rightarrow T^{2} Y$ is the natural projection. The set of all CS on $X \times Y$ is denoted by $\Gamma_{c}^{r}(X \times Y)$ or briefly $\Gamma_{c}^{r}$.

One can show that any $\xi=(F, G) \in \Gamma_{c}^{r}$ is locally represented by a system of ODEs of the form

$$
\dot{x}=y, \quad \dot{y}=g(x, y, u, v), \quad \dot{u}=v, \quad \dot{v}=h(u, v),
$$

where $g, h \in C^{3}$.

We may look at the first two equations of this system as a system controlled by the system of the last two equations.

Define $L \Gamma_{c}^{r}(X \times Y):=\left\{k \xi+l \eta: k, l \in \mathbb{R}, \xi, \eta \in \Gamma_{c}^{r}\right\}$. The set $\Gamma_{c}^{r}$ is not closed with respect to linear operations over $\mathbb{R}$, but the set $L \Gamma_{c}^{r}$ is a vector space over $\mathbb{R}$.

From the definition of $L \Gamma_{c}^{r}$ it follows that the local representative $\xi_{\alpha \beta}$ of $\xi=(F, G) \in L \Gamma_{c}^{r}$ in a local chart $\left(T_{\alpha \times \beta}, \tau_{X \times Y}^{-1}\left(U_{\alpha} \times V_{\beta}\right)\right)$ on $T X \times T Y$ has the form

$$
\dot{x}=k y, \quad \dot{y}=k g_{\alpha \times \beta}(x, y, u, v), \quad \dot{u}=k v, \quad \dot{v}=k h_{\beta}(u, v),
$$

where $k \in \mathbb{R}, g_{\alpha \beta} \in C^{r}\left(\alpha(U) \times \mathbb{R}^{n} \times \beta(V) \times \mathbb{R}^{n}, \mathbb{R}^{n}\right), h_{\beta} \in C^{r}\left(\beta(V) \times \mathbb{R}^{n}, \mathbb{R}^{n}\right)$. In the sequel we identify $\xi_{\alpha \beta}$ with the system (2.6). If $k \neq 0$ then we say that $\xi$ has ordinary structure. Define

$$
\begin{aligned}
\left\|\xi_{\alpha \beta}\right\|_{r}:= & |k|+\sup _{(x, y, u, v) \in K_{\alpha \beta}}\left\{\left\|g_{\alpha \beta}(x, y, u, v)\right\|, \ldots,\left\|d_{(x, y, u, v)}^{r} g_{\alpha \beta}\right\|_{r}\right\} \\
& +\sup _{(u, v) \in L_{\beta}}\left\{\left\|h_{\beta}(u, v)\right\|, \ldots,\left\|d_{(u, v)}^{r} h_{\beta}\right\|_{r}\right\}
\end{aligned}
$$

where $\|.\|_{k}$ is the norm on the space of the corresponding $k$-multilinear mappings, $K_{\alpha \beta}:=\alpha(U) \times \mathbb{R}^{n} \times \beta(V) \times \mathbb{R}^{n}, L_{\beta}=\beta(V) \times \mathbb{R}^{n}$. We identify the vector field $\xi_{\alpha \beta}$ with the mapping $F_{\alpha \beta}: \alpha(U) \times \mathbb{R}^{n} \times \beta(V) \times \mathbb{R}^{n} \rightarrow \mathbb{R}^{4 n}$,

$$
F_{\alpha \beta}(x, y, u, v):=\left(k y, k g_{\alpha \beta}(x, y, u, v), k v, k h_{\beta}(u, v)\right),
$$

and define

$$
\left\|F_{\alpha \beta}\right\|_{r}:=\left\|\xi_{\alpha \beta}\right\|_{r}
$$


Let $\xi \in L \Gamma_{c}^{r},\left\{\left(U_{i}, \alpha_{i}\right)\right\}_{i=1}^{m},\left\{\left(V_{j}, \beta_{j}\right)\right\}_{j=1}^{l}$ be systems of charts on $X$ and $Y$ that cover $X$ and $Y$, respectively, and let $\xi_{i j}$ be the local representative of $\xi$ in the natural chart $\left(T_{\alpha_{i} \times \beta_{j}}, \tau_{X \times Y}^{-1}\left(U_{i} \times V_{j}\right)\right)(i=1, \ldots, m, j=1, \ldots, l)$.

From the definition of $L \Gamma_{c}^{r}$ we see that there is a constant $k$ (independent of $i, j)$ and mappings $g_{i j} \in C^{r}\left(\alpha_{i}\left(U_{i}\right) \times \mathbb{R}^{n} \times \beta_{j}\left(V_{j}\right) \times \mathbb{R}^{n}, \mathbb{R}^{n}\right)$, $h_{j} \in C^{r}\left(\beta_{j}\left(V_{j}\right) \times \mathbb{R}^{n}, \mathbb{R}^{n}\right)$ such that $\xi_{i j}$ is represented by the system

$$
\dot{x}=k y, \quad \dot{y}=k g_{i j}(x, y, u, v), \quad \dot{u}=k v, \quad \dot{v}=k h_{j}(u, v) .
$$

Define $\left\|\xi_{i j}\right\|_{r}$ similarly to (2.8) and let

$$
\|\xi\|_{r}:=\sup \left\{\left\|\xi_{i j}\right\|_{r}: i \in\{1, \ldots, m\}, j \in\{1, \ldots, l\}\right\} .
$$

The vector space $\mathcal{G}_{c}^{r}(X \times Y):=\left\{\xi \in L \Gamma_{c}^{r}(X \times Y):\|\xi\|_{r}<\infty\right\}$ is a Banach space.

REMARK. In all our considerations we assume for simplicity that $\operatorname{dim} X$ $=\operatorname{dim} Y$. However, from the point of view of applications to control problems, it is reasonable to study also the cases $\operatorname{dim} X \neq \operatorname{dim} Y$.

Definition 2.3. Let $A, B, X$ and $Y$ be smooth manifolds. By a parametrized cascade second order $O D E$ (briefly PCS) of class $C^{r}$ on $X \times Y$ (with parameter set $A \times B)$ we mean a couple $\xi=(F, G)$, where $F: T X \times T Y \times$ $A \times B \rightarrow T^{2} \times T^{2}, G: T Y \times B \rightarrow T^{2} Y$ are $C^{r}$-mappings with the following properties:

(1) $\pi_{1} \circ F_{(\dot{y}, a, b)}$ is a second order ODE on $X$ for all $(\dot{y}, a, b) \in T Y \times A \times B$, $F_{(\dot{y}, a, b)}(x):=F(\dot{x}, \dot{y}, a, b)$, i.e. $\pi_{1} \circ F_{\dot{y}}$ is a parametrized second order ODE on $X$ with parameter $(a, b) \in A \times B\left(F_{\dot{y}}(\dot{x}, a, b):=F(\dot{x}, \dot{y}, a, b)\right)$

(2) $G$ is a parametrized second order ODE on $Y$ and $\pi_{2} \circ F(\dot{x}, \dot{y}, a, b)=$ $G(\dot{y}, b)$ for all $(\dot{x}, \dot{y}, a, b) \in T X \times T Y \times A \times B$

$\left(\pi_{1}, \pi_{2}\right.$ are the projections defined in Definition 2.2). The set of all such objects is denoted by $\widetilde{\mathcal{H}}_{c}^{r}(X \times Y, A, B)$.

Throughout this paper we assume that $A, B, X$ and $Y$ are compact.

Sometimes we control a parametrized second order ODE by a second order ODE which is independent of parameters. In this case we have $B=\emptyset$ in Definition 2. 3. The set of all such objects is denoted by $\widetilde{\mathcal{H}}_{c}^{r}(X \times Y, A)$.

Let $L \widetilde{\mathcal{H}}_{c}^{r}(X \times Y, A, B)=\left\{k \xi+l \eta: k, l \in \mathbb{R}, \xi, \eta \in \widetilde{\mathcal{H}}_{c}^{r}(X \times Y, A, B)\right.$ (briefly $L \widetilde{\mathcal{H}}_{c}^{r}$ ).

Let $\xi \in L \widetilde{\mathcal{H}}_{c}^{r},\left\{\left(U_{i}, \alpha_{i}\right)\right\}_{i=1}^{m},\left\{\left(V_{j}, \beta_{j}\right)\right\}_{j=1}^{l}$ be systems of charts on $X$ and $Y$, respectively, as in the definition of $\|\xi\|_{r}$ for $\xi \in L \Gamma_{c}^{r}$ (see (2.10)) and let $\left\{\left(A_{k}, a_{k}\right)\right\}_{k=1}^{p},\left\{\left(B_{s}, b_{s}\right)\right\}_{s=1}^{q}$ be systems of charts on $A$ and $B$ covering $A$ and $B$, respectively. Let $\left(T_{\alpha_{i} \times \beta_{j}}, \tau_{X \times Y}^{-1}\left(U_{i} \times V_{j}\right)\right)$ be the natural chart on $X \times Y$ as in the definition of (2.9) and let $\xi_{i j k s}$ be the local representative of $\xi \in L \widetilde{\mathcal{H}}_{c}^{r}$ in this chart and the above charts on $X$ and $Y$. Then $\xi_{i j k s}$ is 
represented by a parametrized system of differential equations of the form

$$
\dot{x}=k y, \quad \dot{y}=k g_{i j k s}(x, y, u, v, \mu, \nu), \quad \dot{u}=k v, \quad \dot{v}=k h_{j s}(u, v, \nu),
$$

where $x, y, u, v \in \mathbb{R}^{n}, \mu \in \mathbb{R}^{m_{1}}, \nu \in \mathbb{R}^{m_{2}}\left(\operatorname{dim} X=\operatorname{dim} Y=n, \operatorname{dim} A=m_{1}\right.$, $\left.\operatorname{dim} B=m_{2}\right)$.

Define $\left\|\xi_{i j k s}\right\|_{r}$ similarly to (2.8) and let

$\|\xi\|_{r}:=\sup \left\{\left\|\xi_{i j k s}\right\|_{r}: i \in\{1, \ldots, m\}, j \in\{1, \ldots, l\}, k \in\{1, \ldots, p\}\right\}$.

The space

$$
\mathcal{H}_{c}^{r}(X \times Y, A, B):=\left\{\xi \in L \widetilde{\mathcal{H}}_{c}^{r}(X \times Y, A, B):\|\xi\|_{r}<\infty\right\}
$$

is a Banach space.

Let $\left(T^{2} X\right)_{0}$ be the zero section of $T^{2} X$, i.e. $\left(T^{2} X\right)_{0}=\left\{0[\dot{x}] \in T^{2} X\right.$ : $\dot{x} \in T X\}$, where $0[\dot{x}]$ is the zero element of $T_{\dot{x}}(T X)$. The set $\left(T^{2} X\right)_{0}$ is a closed submanifold of $T^{2} X$ diffeomorphic to $T X$ (see [1, pp. 59]). Let $\xi=(F, G) \in \mathcal{H}_{c}^{r}(X \times Y, A, B)$. Then define $C(\xi):=\{(a, b, \dot{x}, \dot{y}) \in A \times B \times$ $\left.T X \times T Y: F(x, y, a, b) \in\left(T^{2} X\right)_{0} \times\left(T^{2} Y\right)_{0}\right\}$.

Proposition 2.4. Let $\xi=(F, G) \in \mathcal{H}_{c}^{r}(X \times Y, A, B)$ have ordinary structure. Then $C(\xi)$ is a subset of $A \times B \times(T X)_{0} \times(T Y)_{0}$, where $(T X)_{0},(T Y)_{0}$ are the zero sections of $T X$ and $T Y$, respectively.

Proof. Let $(a, b, \dot{x}, \dot{y}) \in C(\xi)$, where $\xi=(F, G)$. Then $F_{a b}(\dot{x}, \dot{y}) \in$ $(T X)_{0} \times(T Y)_{0}, G_{b}(\dot{y}) \in(T Y)_{0}$, where $F_{a b}(x, y):=F(\dot{x}, \dot{y}, a, b), G_{b}(\dot{y}):=$ $G(\dot{y}, b)$. Let $\left(T_{\alpha}, \tau_{X}^{-1}\left(U_{\alpha}\right)\right),\left(T_{\beta}, \tau_{Y}^{-1}\left(V_{\beta}\right)\right)$ be natural charts on $T X$ and $T Y$, respectively, such that $\dot{x} \in \tau_{X}^{-1}\left(U_{\alpha}\right), \dot{y} \in \tau_{Y}^{-1}\left(V_{\beta}\right)$. Let $\xi_{a b}:=\left(F_{a b}, G_{b}\right)$ and $\left(\xi_{a b}\right)_{\alpha \beta}$ be the local representative of $\xi_{a b}$ in these charts. If $T_{\alpha}(\dot{x})=(p, q)$ and $T_{\beta}(\dot{y})=(u, v)$ then

$$
\begin{gathered}
\left(\xi_{a b}\right)_{\alpha \beta}(p, q, u, v)=(p, q, k q, k g(p, q, u, v), u, v, k v, k h(u, v)), \\
(p, q) \in \alpha\left(U_{\alpha}\right) \times \mathbb{R}^{n}, \quad(u, v) \in \beta\left(V_{\beta}\right) \times \mathbb{R}^{n}, \quad k \in \mathbb{R} .
\end{gathered}
$$

Since $\xi_{a b}(x, y)=\left(F_{a b}(x, y), G_{b}(y)\right) \in\left(T^{2} X\right)_{0} \times\left(T^{2} Y\right)_{0}$ we have $k q=0$, $k v=0(k \neq 0), g(p, q, u, v)=0$ and $h(u, v)=0$. Thus $T_{\alpha}(\dot{x})=(p, 0)$, $T_{\beta}(\dot{y})=(u, 0)$, i.e. $\dot{x} \in(T X)_{0}, \dot{y} \in(T Y)_{0}$ and the proof is finished.

LEMMA 2.5. Let $Z_{1}$ and $Z_{2}$ be smooth manifolds, $M_{i}$ be a smooth submanifold of $Z_{i}(i=1,2), f \in C^{r}\left(X \times Y, Z_{1}\right), g \in C^{r}\left(X \times Y, Z_{2}\right), r \geq 1$, $F=(f, g) \in C^{r}\left(X \times Y, Z_{1} \times Z_{2}\right)$. Then $F \bar{\pitchfork}\left(M_{1} \times M_{2}\right)(F$ transversally intersects $\left.M_{1} \times M_{2}\right)$ if and only if $f \bar{\pitchfork} M_{1}$ and $g \bar{\pitchfork} M_{2}$.

Proof. By definition of transversality $F \bar{\pitchfork}\left(M_{2} \times M_{2}\right)$ if and only if for any $(x, y) \in T_{x} X \times T_{y} Y=T_{(x, y)}(X \times Y)$,

$$
D F(x, y)\left(T_{(x, y)}(X \times Y)+T_{F(x, y)}\left(M_{1} \times M_{2}\right)=T_{F(x, y)}\left(Z_{1} \times Z_{2}\right),\right.
$$


where $D F(x, y)$ is the derivative of $F$ at $(x, y)$. This equality is equivalent to

$$
\begin{aligned}
D F(x, y)\left(T_{x} X \times T_{y} Y\right)+\left(T_{f(x, y)} M_{1}\right) \times & \left(T_{g(x, y)} M_{2}\right) \\
& =\left(T_{f(x, y)} Z_{1}\right) \times\left(T_{g(x, y)} Z_{2}\right)
\end{aligned}
$$

and this is equivalent to the following system of equalities:

$$
\begin{array}{ll}
\left.D F(x, y)\left(T_{x} X \times T_{y} Y\right)+T_{f(x, y)} M_{1}=T_{f(x, y)} Z_{1} \quad \text { (i.e. } f \bar{\Pi} Z_{1}\right), \\
\left.D g(x, y)\left(T_{x} X \times T_{y} Y\right)+T_{g(x, y)} M_{2}=T_{g(x, y)} Z_{2}, \quad \text { (i.e. } g \bar{\Phi} Z_{2}\right) .
\end{array}
$$

Lemma 2.6. Let $Z_{1}, Z_{2}, M_{1}, M_{2}$ be as in Lemma 2.5, $f \in C^{r}\left(X, Z_{1}\right)$, $g \in C^{r}\left(Y, Z_{2}\right), r \geq 1, F(x, y)=(f(x), g(y))$ for all $(x, y) \in X \times Y$. Assume that $f \bar{\pitchfork} M_{1}$ and $g \bar{\Pi} M_{2}$. Then $F \bar{\hbar}\left(M_{1} \times M_{2}\right)$.

Proof. Define $g \in C^{r}\left(X \times Y, Z_{2}\right), \widetilde{g}(x, y):=g(y)$ for all $(x, y) \in X \times Y$. If $g \bar{\oplus} M_{2}$ then

$$
D g(y)\left(T_{y} Y\right)+T_{g(y)} M_{2}=T_{g(y)} M_{2} .
$$

Since $D g(y)=D_{2} \widetilde{g}(x, y)$ is the partial derivative of $\widetilde{g}$ with respect to $y$, we see from (2.13) that

$$
D_{2} \widetilde{g}(x, y)\left(T_{y} Y\right)+T_{\widetilde{g}(x, y)} M_{2}=T_{\widetilde{g}(x, y)} Z_{2} .
$$

Since $D \widetilde{g}(x, y)\left(T_{x} X \times T_{y} Y\right)=D_{2} \widetilde{g}(x, y)\left(T_{y} Y\right)$, we obtain the equality

$$
D \widetilde{g}(x, y)\left(T_{x} X \times T_{y} Y\right)+T_{\widetilde{g}(x, y)} M_{2}=T_{\widetilde{g}(x, y)} Z_{2},
$$

i.e. $\widetilde{g} \bar{\pitchfork} M_{2}$. One can show analogously that also $\tilde{f} \bar{\Pi} M_{1}$, where $\tilde{f} \in C^{r}(X \times$ $\left.Y, Z_{2}\right), \widetilde{f}(x, y):=f(x)$ for all $(x, y) \in X \times Y$. As a consequence of Lemma 2.5 we obtain $F \bar{\hbar}\left(M_{1} \times M_{2}\right)$.

DEFINITION 2.7.

$$
\begin{aligned}
H_{0}:=\left\{\xi=(F, G) \in \mathcal{H}_{c}^{r}(X \times Y, A, B):\right. & \left.F \bar{\pitchfork}_{(T X)_{0} \times(T Y)_{0} \times A \times B}\left(T^{2} X\right)_{0} \times\left(T^{2} Y\right)_{0}\right\}
\end{aligned}
$$

$\left(f \bar{\pitchfork}_{M} Z\right.$ means that $f$ transversally intersects $Z$ along the set $M$ ).

Proposition 2.8. Suppose that $\operatorname{dim} Y=m_{1}$ and $\operatorname{dim} B=m_{2}$. Then

(1) The set $H_{0}$ is open and dense in $\mathcal{H}_{c}^{r}(X \times Y, A, B), r \geq 1$.

(2) If $\xi \in H_{0}$ then $C(\xi)$ is a compact, $\left(m_{1}+m_{2}\right)$-dimensional $C^{r-1}$ submanifold of $A \times B \times(T X)_{0} \times(T Y)_{0}$.

Proof. Define the mappings

$$
\begin{gathered}
\varrho: \mathcal{H}_{c}^{r}(X \times Y, A, B) \rightarrow C^{r}(T X \times T Y \times A \times B, T X \times T Y), \quad \varrho(F)=F, \\
\operatorname{ev}_{\varrho}: \mathcal{H}_{c}^{r}(X \times Y, A, B) \times T X \times T Y \times A \times \rightarrow T X \times T Y, \\
\operatorname{ev}_{\varrho}(F, \dot{x}, \dot{y}, a, b)=\varrho_{F}(\dot{x}, \dot{y}, a, b):=F(\dot{x}, \dot{y}, a, b) .
\end{gathered}
$$


We shall prove that $\mathrm{ev}_{\varrho} \bar{\Phi}\left(T^{2} X\right)_{0} \times\left(T^{2} Y\right)_{0}$. It suffices to work in coordinates. Locally we have: $T X \approx U \times \mathbb{R}^{n}, T Y \approx V \times R^{n}, A \approx W_{1}, B \approx W_{2}$, $U \subset \mathbb{R}^{n}, V \subset \mathbb{R}^{n}, W_{1} \subset \mathbb{R}^{m_{1}}, W_{2} \subset \mathbb{R}^{m_{2}}$ are open neigbourhoods of the origins, $(T X)_{0} \approx U \times\{0\},(T Y)_{0} \approx V \times\{0\}(\approx$ means locally diffeomorphic). Let $f$ be the local representative of $F$. Then

$$
\begin{gathered}
f: U \times \mathbb{R}^{n} \times V \times W_{1} \times W_{2} \rightarrow \mathbb{R}^{4 n}, \\
f(x, y, u, v, \mu, \nu)=\left(x, y, k y, k f_{1}(x, y, u, v, \mu, \nu), u, v, k v, k f_{2}(u, v, \mu, \nu)\right) .
\end{gathered}
$$

Obviously,

$$
\begin{aligned}
\left(T^{2} X\right)_{0} & \times\left(T^{2} Y\right)_{0} \approx M_{0} \\
& :=\left\{\left(x, y, y_{1}, y_{2}, u, v, z_{1}, z_{2}\right) \in \mathbb{R}^{8 n}: y_{1}=y_{2}=z_{1}=z_{2}=0\right\}=h^{-1}(0),
\end{aligned}
$$

where

$$
h: \mathbb{R}^{8 n} \rightarrow \mathbb{R}^{4 n}, \quad h\left(x, y, y_{1}, y_{2}, u, v, z_{1}, z_{2}\right)=\left(y_{1}, y_{2}, z_{1}, z_{2}\right) .
$$

The map $\operatorname{ev}_{\varrho}$ has its local representative

$$
\left(\mathrm{ev}_{\varrho}\right)_{l}:(f, x, y, u, v, \mu, \nu) \mapsto f(x, y, u, v, \mu, \nu) .
$$

By [6, Proposition 2.76], $\left(\mathrm{ev}_{\varrho}\right)_{l} \bar{\pitchfork} M_{0}$ if and only if the map

$$
h \circ\left(\mathrm{ev}_{\varrho}\right)_{l}:(f, x, y, u, v, \mu, \nu) \mapsto\left(k y, k f_{1}(x, y, u, v, \mu, \nu), k v, k f_{2}(u, v, \nu)\right)
$$

is a submersion. Let us now prove that

$$
P=(f, x, y, u, v, \mu, \nu), \quad \widetilde{P}=(\widetilde{f}, \widetilde{x}, \widetilde{y}, \widetilde{u}, \widetilde{v}, \widetilde{\mu}, \widetilde{\nu}) .
$$

Then the derivative of $h \circ\left(\mathrm{ev}_{\varrho}\right)_{l}$ at $P$ evaluated at $\widetilde{P}$ has the form

$$
\begin{aligned}
d_{P}\left[h \circ\left(\mathrm{ev}_{\varrho}\right)_{l}\right](\widetilde{P}) & =\lim _{s \rightarrow 0} \frac{1}{s}\left[h \circ\left(\mathrm{ev}_{\varrho}\right)_{l}(P+s \widetilde{P})-h \circ\left(\mathrm{ev}_{\varrho}\right)_{l}(P)\right] \\
& =\left(k \widetilde{y}, k d_{U} f_{1}(\widetilde{U})+k \widetilde{f}_{1}(U), k \widetilde{v}, k d_{V} f_{2}(\widetilde{V})+k \widetilde{f}_{2}(V)\right),
\end{aligned}
$$

where $f=\left(f_{1}, f_{2}\right), \widetilde{f}=\left(\widetilde{f}_{1}, \widetilde{f}_{2}\right), U=(x, y, u, v, \mu, \nu), \widetilde{U}=(\widetilde{x}, \widetilde{y}, \widetilde{u}, \widetilde{v}, \widetilde{\mu}, \widetilde{\nu})$, $V=(u, v, \nu), \widetilde{V}=(\widetilde{u}, \widetilde{v}, \widetilde{\nu}), k \neq 0$. We have to show that for any vector $w \in \mathbb{R}^{4 n}$ there exists a $\widetilde{P}=(\widetilde{f}, \widetilde{x}, \widetilde{y}, \widetilde{u}, \widetilde{u}, \widetilde{v}, \widetilde{\mu}, \widetilde{\nu})$ such that

$$
d_{P}\left[h \circ\left(\operatorname{ev}_{\varrho}\right)_{l}(\widetilde{P})\right]=w .
$$

This equation is equivalent to the system

$$
\begin{gathered}
k \widetilde{y}=w_{1}, \quad k \widetilde{v}=v_{3}, \\
k d_{U} f_{1}(\widetilde{U})+k \widetilde{f}_{1}(U)=w_{2}, \\
k d_{V} f_{2}(\widetilde{V})+k \widetilde{f}_{2}(V)=w_{4},
\end{gathered}
$$

where $w=\left(w_{1}, w_{2}, w_{3}, w_{4}\right)$. The first two equations are trivial. If we choose $\widetilde{U}, \widetilde{V}$ arbitrarily then it suffices to find mappings $\widetilde{f}_{1}, \widetilde{f}_{2}$ from the correspond- 
ing spaces with the prescribed values

$$
k \widetilde{f}_{1}(U)=w_{2}-k d_{U} f_{1}(\widetilde{U}), \quad k \widetilde{f}_{2}(V)=w_{4}-k d_{V} f_{2}(\widetilde{U}),
$$

which is a trivial interpolation problem. One can easily check that besides the transversality of the evaluation map $\mathrm{ev}_{\varrho}$ proven above all other assumptions of Abraham's transversality theorems (see [1, Theorem 18.2, 19.1] and also [6]) are satisfied. By these theorems the set $H_{0}:=\{F \in$ $\left.\mathcal{H}_{c}^{r}(X \times Y, A, B): \varrho(F)=F \bar{\pitchfork}_{(T X)_{0} \times(T Y)_{0} \times A \times B}\left[\left(T^{2} X\right)_{0} \times\left(T^{2} Y\right)_{0}\right]\right\}$ is dense in $\mathcal{H}_{c}^{r}(X \times Y, A, B)$. The set $(T X)_{0} \times(T Y)_{0} \times A \times B$ is diffeomorphic to $X \times Y \times A \times B$ and since this is a compact set, the set $H_{0}$ is open. If $F \in H_{0}$ then by [1, Corollary 17.1], $\operatorname{codim} C(\xi)=\operatorname{codim} F^{-1}\left((T X)_{0} \times(T Y)_{0}\right)=$ $\operatorname{codim}\left[(T X)_{0} \times(T Y)_{0}\right]=4 n$, i.e. $\operatorname{dim} C(\xi)=\operatorname{dim}(A \times B)=m_{1}+m_{2}$.

Proposition 2.9. There is an open dense subset $H_{1}$ of $\mathcal{H}_{c}^{r}(X \times Y, A, B)$ such that if $\xi=(F, G) \in H_{1}$ then $C(\xi)$ is a $C^{r-1}$-submanifold of $A \times$ $B \times(T X)_{0} \times(T Y)_{0}$ and $C(G)=\{(b, \dot{y}) \in B \times T Y: G(\dot{y}, b)=0\}$ is a $C^{r-1}$-submanifold of $B \times(T Y)_{0}$, where $\operatorname{codim} C(G)=\operatorname{codim} B=m_{2}$.

Proof. Let $\xi=(F, G) \in H_{0}$, where $H_{0}$ is the set from Proposition 2.8. By Definition 2.3, $\pi_{2} \circ F=G$ and $G(\dot{y}, b)$ is a parametrized second order ODE on $Y$. One can prove as in Proposition 2.4 that $C(G) \subset B \times(T Y)_{0}$. As in the proof of Proposition 2.8 one can show that the set

$$
\widetilde{H}_{0}=\left\{G \in \mathcal{H}_{I I}^{r}(B, Y): G \bar{\pitchfork}_{(T Y)_{0} \times B}\left(T^{2} Y\right)_{0}\right\}
$$

is open and dense in $\mathcal{H}_{I I}^{r}(B, Y)$ which is the set of all parametrized second order ODEs on $Y$ with the parameter set $B$ (see also [3]). Let $H_{1}:=\{\xi=$ $\left.(F, G) \in H_{0}: G \in \widetilde{H}_{0}\right\}$. Obviously,

$$
H_{1}=H_{0} \cap\left\{\xi=(F, G) \in \mathcal{H}_{c}^{r}(X \times Y, A, B): G \bar{\pitchfork} \widetilde{H}_{0}\right\} .
$$

This is obviously an open dense set in $\mathcal{H}_{c}^{r}(X \times Y, A, B)$. If $\xi=(F, G) \in H_{1}$ then $C(\xi)$ is a $C^{r-1}$-submanifold of $A \times B \times(T X)_{0} \times(T Y)_{0}$ (Proposition $2.8)$ and by the same reason as for $C(\xi)$ the set $C(G)=G^{-1}\left(\left(T^{2} Y\right)_{0}\right.$ is a $C^{r-1}$-submanifold of $B \times(T Y)_{0}$ and $\operatorname{codim} C(G)=\operatorname{dim} B=m_{2}$.

Now consider the case when $B=\emptyset$, i.e. the set $\mathcal{H}_{c}^{r}(X \times Y, A)$. If $\xi=(F, G)$ then $G$ is independent of the parameter. From Shahshahani's theorem [9] (see also $[3,4,5]$ ) it follows that the second order ODE $G$ is generically Kupka-Smale, i.e. all its critical elements (critical points and closed orbits) are isolated and hyperbolic. Thus assume that $G$ is Kupka-Smale. Then the set $K(G):=C(G) \cup P(G)$ is finite $(C(G)$ is the set of all critical points of $G$ and $P(G)$ is the set of all periodic orbits of $G$ ) and if $y_{0} \in C(G)$ then we have defined the parametrized second order ODE $\pi_{1} \circ F_{y_{0}}$ on $X \times A$ with parameter set $A$. Now assume that $\operatorname{dim} A=1$. To prove generic results concerning the set $\mathcal{H}_{c}^{r}(X \times Y, A)$ it suffices to apply generic results for 1- 
parameter families of second order ODEs on manifolds proved in $[3,4,5]$ in a slightly modified form. We shall prove the following theorem.

THEOREM 2.10. There exists a residual (i.e. massive by [6]) subset $\mathrm{H}_{2}$ of $\mathcal{H}_{c}^{r}(X \times Y, A)$ such that if $\xi=(F, G) \in \mathcal{H}_{c}^{r}(X \times Y, A)$ then:

(1) $G$ is Kupka-Smale, i.e. the sets $P(G)$ and $C(G)$ of all periodic orbits and critical points of $G$, respectively, are isolated $(C(G)$ is finite) and hyperbolic.

(2) If $y_{0} \in C(G)$ then the 1-parameter family $F_{y_{0}}:(\dot{x}, a) \mapsto F\left(\dot{x}, y_{0}, a\right)$ of second order ODEs is generic in the set $\mathcal{H}^{r}(X, A)$ of all 1-parameter families of second order ODEs on $X$, i.e.:

(a) The set $C\left(F_{y_{0}}\right):=\{(a, \dot{x}) \in A \times T X: \dot{x}$ is a critical point of the vector field $\left.F_{y_{0} a}: \dot{z} \mapsto F_{y_{0}}(\dot{z}, a)\right\}$ is a compact 1-dimensional submanifold of $A \times(T X)_{0}$.

(b) The set $C_{0}\left(F_{y_{0}}\right):=\left\{(a, \dot{x}) \in C\left(F_{y_{0}}\right): \dot{F}_{y_{0} a}(\dot{x})\right.$ is not a surjective map $\}$ is a submanifold of $C\left(F_{y_{0} a}\right)$ of codimension 1 , i.e. of dimension 0 , where $\dot{F}_{y_{0} a}$ is the hessian of $F_{y_{0} a}$ at the critical point $\dot{x}$ (see [1]).

(c) Let $\left(a_{0}, \dot{z}_{0}\right) \in C\left(F_{y_{0}}\right), \dot{z}_{0} \in T_{z_{0}} X,(U, \alpha)$, be a chart on $X, \dot{z}_{0} \in$ $U,(V, \beta)$ be a chart on $A, a_{0} \in V, \mathcal{F}(p, q, \varepsilon)$ be the main part of the local representative of $F_{y_{0}}$ in the chart $(V, \beta)$ and the chart on $T X$ derived from $(U, \alpha)$, where $\alpha\left(a_{0}\right)=\varepsilon_{0}, T_{\alpha}\left(\dot{z}_{0}\right)=\left(p_{0}, q_{0}\right)$, and let $c_{0}=\left(p_{0}, q_{0}, \varepsilon_{0}\right)$. Then

(c1) the map $D\left(a_{0}, \dot{z}_{0}\right) \mathcal{F}_{y_{0}}$ is surjective, i.e.

$$
\operatorname{rank}\left(\frac{\partial \mathcal{F}\left(c_{0}\right)}{\partial p}, \frac{\partial \mathcal{F}\left(c_{0}\right)}{\partial q}, \frac{\partial \mathcal{F}\left(c_{0}\right)}{\partial \varepsilon}\right)=2 n .
$$

(c2) $\left(a_{0}, \dot{z}_{0}\right) \in C_{0}\left(\mathcal{F}_{y_{0}}\right)$ if and only if

$$
\operatorname{rank}\left(\frac{\partial \mathcal{F}\left(c_{0}\right)}{\partial p}, \frac{\partial F\left(c_{0}\right)}{\partial q}\right)=2 n-1 .
$$

(c3) If $\left(a_{0}, \dot{z}_{0}\right) \in C_{0}\left(F_{y_{0}}\right)$ then the charts $(U, \alpha),(V, \beta)$ can be chosen in such a way that $\beta\left(a_{0}\right)=0, T_{\alpha}\left(\dot{z}_{0}\right)=0$, $T_{\alpha}\left(C\left(F_{y_{0}}\right)\right) \cap \tau_{X}^{-1}(U)=\left\{\left(\varepsilon, p_{1}, \ldots, p_{n}, q\right) \in \mathbb{R} \times \mathbb{R}^{n} \times \mathbb{R}^{n}:\right.$ $q=0, \varepsilon=\Phi_{0}\left(p_{1}\right), p_{i}=\Phi_{i}\left(p_{1}\right), i=2, \ldots, n, \varepsilon \in \beta(V)$, $\left.p_{1} \in J\right\}$, where $\Phi_{i}$ are $C^{r}$-functions on an interval $J \subset \mathbb{R}$, $0 \in J, \Phi_{j}(0)=0, j=1, \ldots, n, d \Phi_{0}(0) / d x_{1}=0, d^{2} \Phi_{0}(0) / d x_{1}^{2}$ $>0$. If $\varepsilon=\beta(a)$ then there exists just one couple of points $\left(a, \dot{z}_{1}\right),\left(a, \dot{z}_{2}\right) \in C\left(F_{y_{0}}\right)$ and if $s$ is the number of positive eigenvalues of the map $\dot{F}_{y_{0} a}\left(z_{2}\right)$ then the number of positive eigenvalues of the map $\dot{F}_{y_{0} a}\left(z_{2}\right)$ is either $s+1$ or $s-1$. 
(c4) If $\left(a_{0}, \dot{z}_{0}\right) \in C_{0}\left(F_{y_{0}}\right)$ then the map $\dot{F}_{y_{0} a}\left(\dot{z}_{0}\right)$ has zero eigenvalue of multiplicity 1 .

(d) If $y_{0} \in C(G)$ and $\left(a_{0}, \dot{z}_{0}\right) \in C\left(F_{y_{0}}\right)-C_{0}\left(F_{y_{0}}\right)$ then $\left(a_{0}, y_{0}, \dot{z}_{0}\right)$ is a hyperbolic critical point of $\xi=(F, G)$ and if $\left(a_{0}, \dot{z}_{0}\right) \in C_{0}\left(f_{y_{0}}\right)$ then there is a saddle-node bifurcation of $\xi$ near $\left(a_{0}, y_{0}, \dot{z}_{0}\right)$ (i.e. similar assertions to (2)(a)-(c) hold for the whole vector field $\xi)$.

Pr o of. The assertion (1) is the Kupka-Smale theorem (see e.g. [9]). The assertion (2)(a) is a consequence of Proposition 2.8. For the simplicity of our further considerations we assume $A=\mathbb{R}, X=\mathbb{R}^{n}$, i.e. we are working in coordinates and the corresponding conclusions for the space $\mathcal{H}_{c}^{r}(X \times Y, A)$ can be made in the usual way. We shall work in the space $C_{B}^{r}:=C_{B}^{r}(\mathbb{R} \times$ $\left.\mathbb{R}^{2 n} \times \mathbb{R}^{2 n}, \mathbb{R}^{2 n}\right):=\left\{h \in C^{r}\left(\mathbb{R} \times \mathbb{R}^{2 n} \times \mathbb{R}^{2 n}, \mathbb{R}^{2 n}\right):\|h\|_{r}<\infty\right\}$. In this case we may identify the set $\mathcal{H}_{c}^{r}(X \times Y, A)$ with the set of parametrized systems of differential equations on $\mathbb{R}^{4 n}$ of the form

$$
\xi:= \begin{cases}\dot{p}=k q, & \dot{q}=k f(\varepsilon, p, q, u, v), \\ \dot{u}=k v, & \dot{v}=k g(u, v),\end{cases}
$$

where $k \in \mathbb{R}, f \in C_{b}^{r}, g \in C_{B}^{r}$. The last equation of the system is independent of the parameter and we simply write $g \in C_{B}^{r}$, omitting the number of variables. As above we define the norm $\|\xi\|_{r}:=|k|+\|f\|_{r}+\|g\|_{r}$. Denote the set of all systems of the form (2.16) with the norm $\|\xi\|_{r}<\infty$ by $D^{r}$. The space $\left(D^{r},\|\cdot\|_{r}\right)$ is a Banach space. Let $F_{\varepsilon}(x, y, u, v):=F(\varepsilon, x, y, u, v)$. The set of critical points of $\xi$ is $C(\xi)=\left\{(\varepsilon, x, u, v) \in \mathbb{R} \times \mathbb{R}^{4 n}: y=0, v=0\right.$, $f(\varepsilon, x, y, u, v)=0, g(u, v)=0\}$ and $C_{0}(\xi)=\{(\varepsilon, u, v) \in C(\xi)$ : the matrix of the linearization of $\xi$ at $(\varepsilon, x, u, v)$ is singular $\}$.

Now let $J$ be the set of all 1-jets of mappings from $C_{B}^{r}$ and $J_{I I}^{1}:=\left\{j^{1} \xi \in\right.$ $\left.J^{1}: \xi \in D^{r}\right\}$. If $\xi$ is of the form (2.16) then

$$
\begin{array}{r}
j^{1} \xi(\varepsilon, p, q, u, v)=(\varepsilon, p, q, k q, k f(\varepsilon, p, q, u, v), u, v, \\
k v, k g(u, v), B(\varepsilon, p, q, u, v)),
\end{array}
$$

where

$$
B=k\left(\begin{array}{ccccc}
0 & 0 & I & 0 & 0 \\
a & A_{0} & B_{0} & C_{0} & D_{0} \\
0 & 0 & 0 & 0 & I \\
0 & 0 & 0 & P_{0} & Q_{0}
\end{array}\right)
$$

with $a=\partial f(c) / \partial \varepsilon, A_{0}=\partial f(c) / \partial q, C_{0}=\partial f(c) / \partial u, D_{0}=\partial f(c) / \partial v, P_{0}=$ $\partial g(d) / \partial u, Q_{0}=\partial g(d) / \partial v, c=(\varepsilon, p, q, u, v), d=(u, v)$. Then $J_{I I}^{1}$ is obviously a linear subspace of $J^{1}$. Define the sets

$$
M_{I I}^{1}=\left\{\left(\varepsilon, p, q, q_{1}, u, v, v_{1}, B\right) \in \mathbb{R} \times \mathbb{R}^{4 n} \times M(4 n, 4 n+1):\right.
$$

$B$ is a matrix of the form $(2.17)\}$, 


$$
\left(M_{I I}^{1}\right)_{0}=\left\{\left(\varepsilon, p, q, q_{1}, u, v, v_{1}, B\right) \in M_{I I}^{1}: q_{1}=0, v_{1}=0, \operatorname{det} B=0\right\} .
$$

Then $M_{I I}^{1}$ is obviously a linear subspace of $\mathbb{R} \times \mathbb{R}^{6 n} \times M(4 n, 4 n+1)$ and $\left(M_{I I}^{1}\right)_{0}$ is an algebraic manifold. By the Whitney stratification theorem (see e.g. [1] or [6]) there is an ordered Whitney stratification $\left(M_{I I}^{1}\right)_{0}=\bigcup_{i=1}^{m} M_{i}$, where $M_{i}, i=1, \ldots, m$, are smooth manifolds. Let $X_{n}:=\mathbb{R} \times \mathbb{R}^{6 n}$. Define the map $\varrho_{1}: D^{r} \rightarrow C^{r-1}\left(X_{n}, J_{I I}^{1}\right), \varrho(\xi)=j^{1} \xi$, where $j^{1} \xi$ is the 1 -jet extension of $\xi$. This map is a $C^{r-1}$-representation. Let us check that $\mathrm{ev}_{\varrho} \bar{\hbar}\left(M_{I I}^{1}\right)_{0}$ (i.e. $\left.\quad \mathrm{ev}_{\varrho} \bar{\hbar} M_{i}, i=1, \ldots, m\right)$, where $\mathrm{ev}_{\varrho}: D^{r} \times J_{I I}^{1},(\xi, \varepsilon, p, q, u, v) \mapsto$ $\varrho(\xi)(\varepsilon, p, q, u, v)$. We shall prove that ev $\varrho 币 Z$ for any submanifold $Z$ of $M_{I I}^{1}$.

Let $w=\operatorname{ev}_{\varrho}(\xi, c) \in Z$, where $\xi \in D^{r}, c=(\varepsilon, p, q, u, v) \in X_{n}$. Then $\operatorname{ev}_{\varrho} \bar{T}_{(\xi, c)} Z$ if and only if

$$
D_{(\xi, c)} \operatorname{ev}_{\varrho}\left(D^{r} \times X_{n}\right)+T_{w} Z=T_{w} M_{I I}^{1} .
$$

It suffices to prove that $D_{(\xi, c)} \mathrm{ev}_{\varrho}$ is surjective. If $(\xi, c),(\widetilde{\xi}, \widetilde{c}) \in D^{r} \times X_{n}$ then

$$
D_{(\xi, c)} \operatorname{ev}_{\varrho}(\widetilde{\xi}, \widetilde{c})=\left(\widetilde{c}, D_{c} \xi(\widetilde{c})+\widetilde{\xi}(c), D_{c}^{2} \xi(\widetilde{c})+D_{c} \widetilde{\xi}\right) .
$$

Since $T_{w} M_{I I}^{1}$ can be identified with $M_{I I}^{1}$, it suffices to prove that for any $\omega=(\kappa, \widehat{p}, \widehat{q}, \widehat{u}, \widehat{v}, U, \widetilde{B}) \in M_{I I}^{1} \equiv T_{w} M_{I I}^{1}$ there exist $(\widetilde{\xi}, \widetilde{c}) \in D^{r} \times X_{n}$ such that $D_{(\xi, c)} \operatorname{ev}_{\varrho}(\widetilde{\xi}, \widetilde{c})=\omega(U$ corresponds to a value of the vector field and $\widetilde{B}$ has the form (2.17), where in (2.17) there is a tilde above the letters $\left.A_{0}, B_{0}, C_{0}, D_{0}, P_{0}, Q_{0}\right)$. It suffices to choose $\varepsilon=\kappa, \widetilde{c}=\widehat{c}$ and $\widetilde{\xi}$ such that $\widetilde{\xi}(\widetilde{c})=U-D_{c} \xi, D_{c} \widetilde{\xi}=\widetilde{B}-D_{c}^{2} \xi(\widetilde{c})$. One can easily check that these equalities can be satisfied by a suitable choice of $\widetilde{\xi}$ with prescribed values (and the values of its derivatives) at the point $\widetilde{c}$. This is a trivial interpolation problem. All other assumptions of Abraham's transversality theorem are also satisfied. By this theorem for any compact neighbourhood $K$ of $c_{0}$ the set $D_{0}^{r}:=\left\{\xi \in D^{r}: \xi \bar{\pitchfork}_{K}\left(M_{I I}^{1}\right)_{0}\right\}$ is open and dense in $D^{r}$. Since codim $M_{i}=$ $2 n+1$ and codim $M_{i}>2 n+1$ for $i>1$, from [1, Corollary 17.2] it follows that $\operatorname{codim} C_{0}(\xi) \cap K=\operatorname{codim}(\varrho(\xi))^{-1}\left(M_{1}\right) \cap K=\operatorname{codim} M_{1}=2 n+1$, i.e. $\operatorname{dim} C_{0}(\xi) \cap K=0$. Let $\xi \in D_{0}$ and $c_{0}=\left(\varepsilon_{0}, p_{0}, q_{0}, u_{0}, v_{0}\right) \in C_{0}(\xi) \cap K$ and let $d_{c_{0}} \xi$ be the derivative of the right-hand side of (2.16) at $c_{0}$. Since $\varrho(\xi)\left(c_{0}\right) \in M_{1}$, corank $d_{c_{0}} \xi=1$. The derivative $d_{c_{0}} \xi$ is of the form (2.17). By (a) we have

$$
\operatorname{det}\left(\begin{array}{cc}
0 & I \\
P_{0} & Q_{0}
\end{array}\right) \neq 0 \quad \text { (generically) }
$$

This yields that

$$
\operatorname{rank}\left(\begin{array}{ccccc}
0 & 0 & I & 0 & 0 \\
a & A_{0} & B_{0} & C_{0} & D_{0}
\end{array}\right)=2 n
$$


We shall show that generically

$$
\operatorname{rank}\left(\begin{array}{ccc}
0 & 0 & I \\
a & A_{0} & B_{0}
\end{array}\right)=2 n .
$$

Define the map

$$
\sigma(\xi)_{\left(u_{0}, v_{0}\right)}:(\varepsilon, p, q) \mapsto\left(k q, k f\left(\varepsilon, p, q, u_{0}, v_{0}\right)\right) .
$$

Let $\widetilde{J}^{1}$ be the set of all 1-jets of mappings from the set $C_{B}^{r}\left(\mathbb{R} \times \mathbb{R}^{n}, \mathbb{R}^{n}\right)$ and $\widetilde{J}_{I I}^{1}\left(u_{0}, v_{0}\right)=\left\{j^{1} \sigma(\xi)_{\left(u_{0}, v_{0}\right)}: \xi \in D^{r}\right\}$, where $\sigma(\xi)_{\left(u_{0}, v_{0}\right)}$ is defined by $(2.21)$. Obviously,

$$
j^{1} \sigma(\xi)_{\left(u_{0}, v_{0}\right)}(\varepsilon, p, q)=\left(\varepsilon, p, q, f\left(\varepsilon, p, q, u_{0}, v_{0}\right), \widetilde{B}\left(\varepsilon, p, q, u_{0}, v_{0}\right)\right),
$$

where $\widetilde{B}$ is the matrix from (2.20) with elements described in (2.17). Define the sets

$$
\begin{gathered}
\widetilde{M}_{I I}^{1}=\left\{\left(\varepsilon, p, q, q_{1}, \widetilde{B}\right) \in \mathbb{R} \times \mathbb{R}^{3 n} \times M(2 n, 2 n+1):\right. \\
\widetilde{B} \text { is the matrix of the form }(2.20)\}, \\
\left(\widetilde{M}_{I I}^{1}\right)_{0}=\left\{\left(\varepsilon, p, q, q_{1}, B\right) \in \widetilde{M}_{I I}^{1}: q=0, q_{1}=0, \operatorname{det} \widetilde{B}=0\right\} .
\end{gathered}
$$

Let $\left(\widetilde{M}_{I I}^{1}\right)_{0}=\bigcup_{i=1}^{s} \widetilde{M}_{i}$ be the ordered Whitney stratification. Then codim $\widetilde{M}_{i}$ $=2 n+1$. Let $\widetilde{X}_{n}=\mathbb{R} \times \mathbb{R}^{3 n}$ and

$$
\widetilde{\varrho}: D^{r} \rightarrow C^{r-1}\left(\widetilde{X}_{n}, \widetilde{J}_{I I}^{1}\left(u_{0}, v_{0}\right)\right), \quad \widetilde{\varrho}(\xi)=j^{1} \sigma(\xi)_{\left(u_{0}, v_{0}\right)} .
$$

This map is a $C^{r-1}$-representation and one can check as for the map $\varrho$ above that ev $\widetilde{\varrho} \bar{\Pi} \widetilde{Z}$ for any submanifold $\widetilde{Z}$ of $\widetilde{J}_{I I}^{1}\left(u_{0}, v_{0}\right)$. From Abraham's transversality theorem it follows that

$$
D_{00}^{r}=\left\{\xi \in D^{r}: \widetilde{\varrho}(\xi)=j^{1} \sigma(\xi)_{\left(u_{0}, v_{0}\right)} \bar{\pitchfork}_{K}\left(\widetilde{M}_{I I}^{1}\right)_{0}\right\}
$$

is open and dense in $D_{0}^{r}$, where $K \subset \mathbb{R} \times \mathbb{R}^{2 n}$ is a compact set. From [1, Corollary 17.2] we see that if $\xi \in D_{00}^{r}$ then $\operatorname{codim} C_{0}\left(\sigma(\xi)_{\left(u_{0}, v_{0}\right)}\right) \cap K=$ $2 n+1$, where $C_{0}\left(\sigma(\xi)_{\left(u_{0}, v_{0}\right)}\right)=\widetilde{\varrho}(\xi)^{-1}\left(\widetilde{M}_{1}\right)$, i.e. $\operatorname{dim} C_{0}\left(\sigma(\xi)_{\left(u_{0}, v_{0}\right)}\right) \cap K=$ 0 . If $\widetilde{c}_{0}=\left(\varepsilon_{0}, p_{0}, q_{0}\right) \in C_{0}\left(\sigma(\xi)_{\left(u_{0}, v_{0}\right)}\right)$ then the transversality condition, $j^{1} \sigma(\xi)_{\left(u_{0}, v_{0}\right)} \bar{\pitchfork}_{K}\left(M_{I I}^{1}\right)_{0}$, yields $(2.20)$, where

$$
\operatorname{rank}\left(\begin{array}{cc}
0 & I \\
A_{0} & B_{0}
\end{array}\right)=2 n-1,
$$

i.e. the matrix from this equality has zero eigenvalue of multiplicity 1 . One can write $\xi \in D_{00}^{r}$ near the point $\left(\varepsilon, p_{0}, q_{0}\right)$ in the form

$$
\dot{p}=k q, \quad \dot{q}=k f\left(\varepsilon, p, q, u, u_{0}, v_{0}\right) .
$$

Without loss of generality we may assume that $k=1$ (if not, it is possible to achieve it by a transformation of time). This system can be written in 
the form

$$
\dot{p}=q, \quad \dot{q}=A(\varepsilon) p+B(\varepsilon) q+h(\varepsilon, p, q),
$$

where $A, B, h \in C^{r}, h(0, p, q)=o(\|(p, q)\|)$ (we omit $u_{0}, v_{0}$ in the notation). Let $\widetilde{\mathcal{C}}=\operatorname{diag}\{C, C\}$, where $C \in M(n)$ is a regular matrix and let

$$
\mathcal{A}(\varepsilon)=\left(\begin{array}{cc}
0 & I \\
A(\varepsilon) & B(\varepsilon)
\end{array}\right) .
$$

Obviously,

$$
\widetilde{C} \mathcal{A}(0) \widetilde{C}^{-1}=\left(\begin{array}{cc}
0 & I \\
C A(0) C^{-1} & C B(0) C^{-1}
\end{array}\right) .
$$

Generically the matrix $A(\varepsilon)$ has zero eigenvalue of multiplicity 1 for $\varepsilon=0$ and by [6, Lemma 3.65] one can find the matrix $C(\varepsilon)$ in such a way that it is $C^{r}$-differentiable on an open interval $I$ containing $0, C(0)=C$ and the matrix $\widetilde{A}(\varepsilon):=C(\varepsilon) A(\varepsilon)(C(\varepsilon))^{-1}$ is in Jordan's canonical form for all $\varepsilon \in I$. If $p=(C(\varepsilon))^{-1} X$ and $q=(C(\varepsilon))^{-1} Y$ then (2.23) becomes

$$
\dot{X}=Y, \quad \dot{Y}=\widetilde{A}(\varepsilon) X+\widetilde{B}(\varepsilon) Y+\widetilde{h}(\varepsilon, X, Y),
$$

where $\widetilde{A}(\varepsilon)=\operatorname{diag}\{0, \widehat{A}(\varepsilon)\}, \widehat{A}(\varepsilon) \in M(n-1, n-1)$,

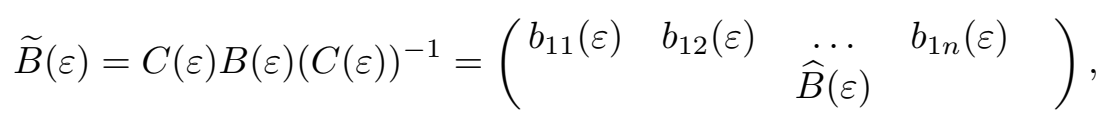

$\widehat{B}(\varepsilon) \in M(n-1, n-1), \widetilde{h}(\varepsilon, X, Y)=C(\varepsilon) h\left(\varepsilon, C(\varepsilon)^{-1} X, C(\varepsilon)^{-1} Y\right)$. The system $(2.26)$ can be written in the form

$$
\begin{aligned}
& \dot{X}=Y, \\
& \dot{Y}_{1}=\alpha \varepsilon+\beta X_{1}^{2}+\widetilde{b}_{11} Y_{1}+\ldots+\widetilde{b}_{1 n} X_{n}+h_{1}\left(\varepsilon, X_{1}, \widehat{X}, Y\right), \\
& \dot{\hat{Y}}=\dot{\widehat{A}}(\varepsilon) \widehat{X}+B(\varepsilon) \widehat{Y}+h_{2}\left(\varepsilon, X_{1}, X, Y\right),
\end{aligned}
$$

where $h_{1}, h_{2} \in C^{r}, X=\left(X_{1}, \ldots, X_{n}\right), \widehat{Y}=\left(Y_{2}, \ldots, Y_{n}\right), \widehat{X}=\left(X_{2}, \ldots\right.$ $\left.\ldots, X_{n}\right), h_{1}\left(\varepsilon, X_{1}, 0, \ldots, 0\right)$ contains terms of order higher than 2 only and $h_{2}(\varepsilon, X, Y)$ contains terms of order higher than 1 only. We denote the vector field $(2.27)$ by $\widetilde{F}$. The set $C(\widetilde{F})$ of all critical points of $\widetilde{F}$ is given by the equalities

$$
\begin{aligned}
& Y=0, \\
& \alpha \varepsilon+\beta X_{1}^{2}+\widetilde{b}_{11}(\varepsilon) Y_{1}+\ldots+\widetilde{b}_{1 n} Y_{n}+h_{1}\left(\varepsilon, X_{1}, \widehat{X}, Y\right)=0, \\
& \widehat{A}(\varepsilon) \widehat{X}+\widehat{B}(\varepsilon) \widehat{Y}+h_{2}\left(\varepsilon, X_{1}, \widehat{X}, Y\right)=0 .
\end{aligned}
$$

Since $\operatorname{det} \widehat{A}(0) \neq 0$, from the implicit function theorem it follows that there exists a $C^{r}$-map $\widehat{X}=\Psi\left(\varepsilon, X_{1}\right)$ such that $\Psi(0,0)=0$ and

$$
P\left(\varepsilon, X_{1}\right):=\widehat{A}(\varepsilon) \Psi\left(\varepsilon, X_{1}\right)+h_{2}\left(\varepsilon, X_{1}, \Psi\left(\varepsilon, X_{1}\right), 0\right)=0
$$


for $\left(\varepsilon, X_{1}\right)$ from some neighbourhood of $(0,0)$. Let $F$ be the vector field (2.26). Then $d_{0} \widetilde{F}=C\left(d_{0} F\right) C^{-1}$ and therefore the rank condition (2.22) implies that $\alpha \neq 0$. This enables us to use the implicit function theorem by which there exists a $C^{r}$-function $\varepsilon=\Phi_{0}\left(X_{1}\right)$ such that $\Phi_{0}(0)=0$ and $P\left(\Phi_{0}\left(X_{1}\right), X_{1}\right)=0$ in a neighbourhood of the point $X_{1}=0$. From the last equality and from (2.27) we obtain $\Phi_{0}^{\prime}(0)=0$ and $\Phi_{0}^{\prime \prime}(0)=2 \beta$. If $\beta \neq 0$ and $\alpha / \beta<0$ then $\Phi_{0}^{\prime \prime}(0)>0$ and if $\alpha / \beta<0$ then using the change of coordinates $\varepsilon \rightarrow-\varepsilon$ one can obtain again the case $\Phi_{0}^{\prime \prime}(0)>0$. Therefore it suffices to prove that $\beta \neq 0$ generically.

We know that the matrix of linearization of (2.27) with $\varepsilon=0$ at the origin does not have the maximal rank and that its rank is $2 n-1$ (see $(2.22))$. However, any vector field $\xi \in D_{00}^{r}$ satisfies the transversality condition $j^{1} \sigma(\xi)_{\left(u_{0}, v_{0}\right)} \bar{\Pi}\left(M_{I I}^{1}\right)_{0}$. Since the system (2.27) is a local representative of a component of such a vector field this transversality condition is satisfied for this local representative. One can check that this condition implies that the system of equations

$$
\begin{gathered}
\frac{\partial g(\varepsilon, z)}{\partial \varepsilon} q=w_{1}, \\
\frac{\partial^{2} g(\varepsilon, z)}{\partial X_{1}^{2}} p+\frac{\partial^{2} g(\varepsilon, z)}{\partial X_{1} \partial \varepsilon} q=w_{2}
\end{gathered}
$$

is solvable for any $w_{1}, w_{2} \in \mathbb{R}$, where $p, q \in \mathbb{R}$ are unknowns, $z=\left(X_{1}, \widehat{X}, Y\right)$ and $g(\varepsilon, z)$ is the right-hand side of the second equation of $(2.27)$. It is obvious that this system is solvable if and only if $\partial^{2} g(\varepsilon, z) / \partial X_{1}^{2}=\beta \neq 0$ (we know that $\partial g(0,0) / \partial \varepsilon=\alpha \neq 0$ ). The function $\Phi_{0}$ is a solution of the equation (2.28) which can be solved by using the implicit function theorem. Since $\alpha \neq 0, \beta \neq 0$ the function $\Phi_{0}$ has the properties as in (c3). The rest of the assertion (c) and the assertion (d) are consequences of the rank condition (2.15) and the form of the system (2.27). The proof is finished.

\section{References}

[1] R. Abraham and J. Robbin, Transversal Mappings and Flows, W. A. Benjamin, New York, 1967.

[2] M. Medved̆, Oscillatory properties of some classes of nonlinear differential equations, Math. Bohemica 117 (1992), 95-107.

[3] - Generic bifurcations of second order ordinary diferential equations on differentiable manifolds, Math. Slovaca 27 (1977), 9-24.

[4] - Generic bifurcations of second order ordinary differential equations near closed orbits, J. Differential Equations 36 (1980), 98-107.

[5] -, On generic bifurcations of second order ordinary differential equations near closed orbits, Astérisque 50 (1977), 23-29. 
[6] M. Medved, Fundamentals of Dynamical Systems and Bifurcation Theory, Adam Hilger, Bristol, 1992.

[7] —, A class of vector fields on manifolds containing second order ODEs, Hiroshima Math. J. 26 (1996), 127-149.

[8] P. Seibert and R. Suarez, Global stabilization of nonlinear cascade systems, Systems Control Lett. 14 (1990), 347-352.

[9] S. Shahshahani, Second order ordinary differential equations on differentiable manifolds, in: Global Analysis, Proc. Sympos. Pure Math. 14, Amer. Math. Soc., 1970, 265-272.

Department of Mathematical Analysis

Faculty of Mathematics and Physics

Comenius University

Mlynská dolina

84215 Bratislava, Slovakia

E-mail: medved@center.uniba.sk

Reçu par la Rédaction le 23.1.1997

Révisé le 1\%.7.1997 